\title{
Design and Application of a Cloud Logistics Information Management System
}

\author{
Zichen Zhang \\ School of Management, Wuhan University of Technology, Wuhan, China
}

Keywords: logistics industry; cargo positioning; APP.

\begin{abstract}
With the era of more and more intelligence and the rapid development of mobile Internet, the demand for new products in various industries is also increasing. In the logistics industry, the traditional form of logistics can no longer satisfy everyone. The traditional logistics industry has gradually migrated from the PC-side Internet to the mobile Internet. Therefore, the logistics industry has a system that can reasonably locate cargo information in an all-round way and integrate it with it. The APP is in urgent need. Based on this, this article conducts preliminary research and design on this type of information display system.
\end{abstract}

\section{Design background}

In recent years, with the development of the e-commerce industry, more and more users have begun to choose to shop online, and the market demand in the logistics industry will increase dramatically. However, traditional logistics cannot adapt to the convenience of Internet e-commerce, so logistics companies urgently need to transform and upgrade, and developing APP applications is undoubtedly the most reasonable choice. Therefore, only when logistics companies develop APP applications can they bring users a more convenient shopping and receiving experience, and logistics companies will develop better.

The multi-frequency, small batch delivery and timely delivery of customer logistics requirements in the logistics transportation process have greatly increased the frequency of goods delivery, and the delivery time regulations have become more and more strict. In this environment, some large-scale road transportation companies in developed countries have established complete vehicle dispatching systems using communication satellites, GPS, and digital traffic maps. However, due to the large number of logistics companies and the inconsistent working system in China, temporarily There is no more reasonable logistics information management system, and this article designs this information management system based on business management thinking and cloud server technology, hoping to improve the overall quality of the current logistics industry to a certain extent.

\section{Overall design}

The logistics information management system with business management thinking as the main design concept is a trinity interactive system based on cloud, users, and couriers

Based on the various existing simple logistics information display systems, cargo order number query systems, etc., this system combines the original technology and creatively displays vehicle information and geographic information in the supporting APP, adding a certain interactive system , To ensure the user's understanding of the current situation of the goods, to ensure the immediacy and authenticity of logistics information, the general workflow is as follows: 


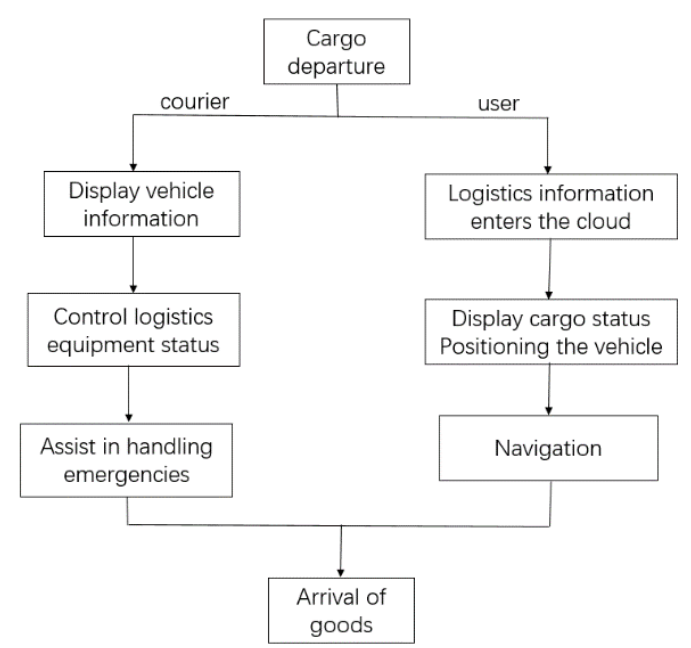

Fig.1 work flow chart

\section{3. module design}

\subsection{Menu module}

After successfully logging in, the user will automatically enter the system homepage, which is the menu module. The menu module is the core of the entire system. After entering the system homepage, users can see detailed information about the entire system. Users can select the main menu and sub-menus they need to enter from the system homepage to complete the user's work needs faster and more efficiently.

The system homepage carries the operating instructions of the entire system. You can see all the submenus of the system by successfully logging into the system and entering the system homepage. Operators only need to click to enter the submenu they need to enter the corresponding interface and proceed to the next step.

\subsection{Positioning module}

The user enters the start tracking interface through the system-related instructions, and understands the relevant information through the unified screen of the system. The user can obtain the relevant operation steps of the start tracking from the system according to his own needs, and follow the system prompts to set related functions. It can complete its own related operation requirements more quickly.

Through the window, the user can intuitively understand the specific content to be tracked, and set the corresponding functions in the page according to actual needs.

\subsection{User Management Module}

The user management module is the most important part of the business management thinking. Through the interface displayed by the system, the user can perform the corresponding user management function operation. According to the relevant operation plan, the user can set the correct operation function to facilitate the smooth operation of the system. The details are shown in the figure below: 


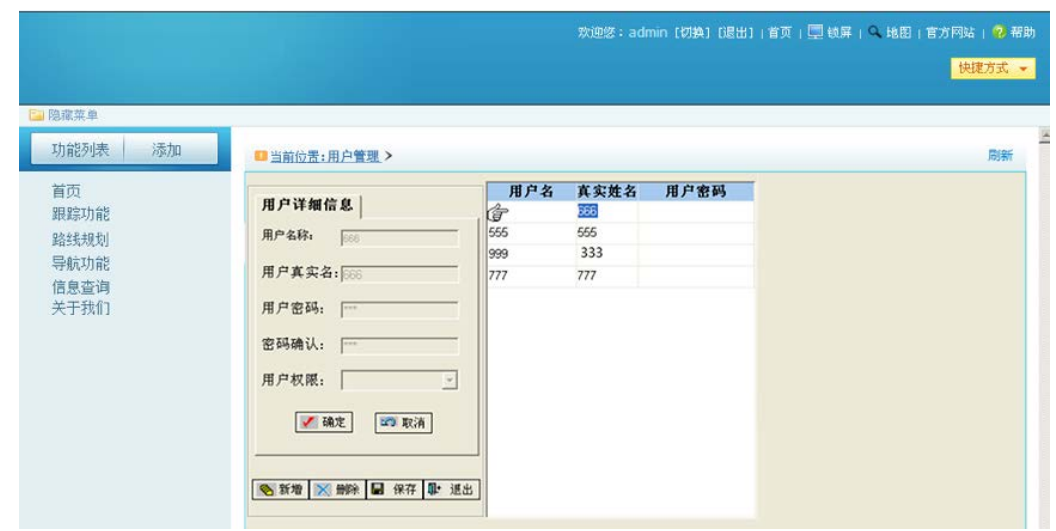

Fig.2 Operation diagram

According to the relevant process operation, the user can know the operation process of the user management function. According to the actual operation situation, the corresponding operation setting can be completed, so that the user can perform the function operation on the page, thus ensuring the integrity of the entire logistics information management system.

\section{Application prospects}

With the development of the Internet, e-commerce business has shown a spurt of growth, and the development of the logistics industry is also gaining momentum. Under such circumstances, the development of various logistics management systems and APPs is a very meaningful thing. This is because through the application of related systems and APPs, logistics companies can better serve users, improve user satisfaction, enhance corporate competitiveness, and ease the relationship between users and logistics workers, and ensure the stability and health of the entire logistics industry development of.

\section{Summary}

With the sharp increase in user demand, more and more logistics companies will rise, so that the competition between them will become more intense. Under such circumstances, in order to survive and develop, logistics companies have to fight for users. The development of logistics information management work systems and their APP applications can better serve users and increase effectively. The competitiveness of enterprises makes enterprises more easily favored and recognized by users. This article puts forward certain ideas and ideas for the design of new systems, hoping to help the logistics industry to make greater progress in the future development.

\section{References}

[1] Liu Xiaona, Wang Zhangfeng. Research on the Training Mode of Applied Innovative Talents in Colleges and Universities[J]. Science Education Journal, 2019(4): 32-33.

[2] Zhang Dongyu, Wen Yafang, Yang Rui, Liu Meijing, Guo Sixu. Talking about the application of big data in logistics management. "The Era of Wisdom", May 2018.

[3] Hu Ying. On the application of information technology in logistics management [J]. Modern Marketing (Late Period), 2018(07): 121.

[4] Gao Mei. Thinking about "Internet +" on warehousing logistics management[J]. Modern Business, 2018(16):47-48 